\title{
Conformational Analysis of Chain Molecules in Liquid Crystalline Phases by a Rotational Isomeric State Scheme with Maximum Entropy Method I. ${ }^{1} \mathrm{H}^{-1} \mathrm{H}$ Dipolar Couplings from $n$-Alkanes Dissolved in a Nematic Solvent
}

\author{
Yuji SASANUMA ${ }^{\dagger}$ \\ Department of Materials Technology, Faculty of Engineering, Chiba University, \\ 1-33 Yayoi-cho, Inage-ku, Chiba 263-8522, Japan
}

(Received May 17, 2000; Accepted June 21, 2000)

\begin{abstract}
For conformational analysis of chain molecules incorporated in nematic fields, a rotational isomeric state (RIS) scheme was revised for use with the maximum entropy method. Analysis thus based proceeds as follows. 1) All possible conformations are enumerated within the RIS approximation. 2) The principal axes of inertia, determined for each conformer, are considered molecular axes, and orientational order parameters are evaluated from dimensions of a rectangular parallelepiped closely fitted to the conformer. 3) Conformational statistical weight factors of first- and second-order interactions are adjusted and order parameters are scaled so as to reproduce experimental observation and maximize the information entropy regarding conformer populations. By this method, ${ }^{1} \mathrm{H}^{-1} \mathrm{H}$ dipolar couplings observed from $n$-hexane, $n$-heptane, $n$-octane, $n$-nonane, and $n$-decane dissolved in a nematic liquid crystal Kodak EK11650 p-pentylphenyl-2-chloro(4-benzylbenzoyloxy)-benzoate [M. E. Rosen, S. P. Rucker, C. Schmidt, and A. Pines, J. Phys. Chem. 97, 3858 (1993)] were analyzed. For all $n$-alkanes, good agreement between theory and experiment was attained. Anisotropic conformers were slightly more populated than in the free state. The present results were quantitatively comparable to those of analysis using the mean-field theory.
\end{abstract}

KEY WORDS $n$-Alkanes / Conformation / Liquid Crystal $/{ }^{1} \mathrm{H}^{-1} \mathrm{H}$ Dipolar Coupling / Rotational Isomeric State Scheme / Maximum Entropy Method /

In the free state, $n$-alkane chains are flexible and the $\mathrm{C}-\mathrm{C}$ bonds prefer the trans to gauche state. Within the rotational isomeric state (RIS) approximation, the conformations and average shapes can be estimated using only two conformational energies $E_{\sigma}$ and $E_{\omega}{ }^{1}$ The former, due to first-order interaction (between $\mathrm{CH}_{2}\left(\right.$ or $\mathrm{CH}_{3}$ ) groups separated by three bonds) is $0.5 \mathrm{kcal} \mathrm{mol}^{-1}$, and the latter, due to second-order interaction (by four bonds) in the $\mathrm{g}^{ \pm} \mathrm{g}^{\mp}$ conformations, is $2.0-3.0 \mathrm{kcal}$ $\mathrm{mol}^{-1}{ }^{2}$ Alkyl chains are found in spacers and tails of thermotropic liquid crystals (LCs), main-chain and sidechain liquid crystalline polymers, surfactants and biological membranes. To understand the phase behavior of these LCs and estimate thermodynamic quantities related to phase transition, the conformations and orientations in the liquid crystalline phases should be clarified.

Nuclear magnetic resonance (NMR) data such as ${ }^{1} \mathrm{H}^{-}$ ${ }^{1} \mathrm{H}$ dipolar couplings and ${ }^{2} \mathrm{H}$ quadrupolar splittings from alkyl chains in the liquid crystalline phases have been analyzed using theoretical models to investigate orientational ordering. ${ }^{3,4}$ The mean-field theory, ${ }^{5}$ based on statistical mechanics, has been used, and the partition function may be calculated from internal and external energies. The former depends only on conformation, and is given as a function of $E_{\sigma}$ and $E_{\omega}$. The latter depends on conformation and orientation. Orientational potential has often been approximated by second-order Legendre polynomials, ${ }^{6,7}$ and much effort has been directed to improvement of the potential function. ${ }^{8-11}$

In previous studies ${ }^{12-14}$ the RIS scheme, developed mainly for polymeric chains, was extended to conforma- tional analysis of chain molecules dissolved in nematic solvents. To reduce the number of adjustable parameters in the simulation, cylindrical-symmetry approximation and single-ordering-matrix approximation were assumed. In the former, biaxiality $S_{X X}-S_{Y Y}$ is neglected, and the latter means the orientational order parameter $S_{Z Z}$ is identical for all the possible conformers. By this method, we analyzed ${ }^{2} \mathrm{H}$ NMR quadrupolar splittings ${ }^{15}$ observed from $n$-alkanes dissolved in nematic solvents as well as ${ }^{1} \mathrm{H}^{-1} \mathrm{H}$ dipolar couplings ${ }^{16,17}$ from $n$-hexane in a nematic LC. ${ }^{12}$ Conformer populations thus evaluated are compatible with molecular rigidity predicted by thermodynamics studies, ${ }^{18,19}$ but have large discrepancy with those determined by mean-field approaches. ${ }^{20}$

This study refines our simulation scheme. From shape anisotropy of the conformer, orientational order parameters $S_{Z Z}$ and $S_{X X}-S_{Y Y}$ were determined for each conformer. The maximum entropy (MaxEnt) method ${ }^{21,22}$ was introduced into the fitting procedure to obtain the most probable results from limited number of experimental data. Even when the parameters outnumber the data, MaxEnt derives the most reliable conclusion.

In this study, using modified methodology, we analyzed (1) ${ }^{1} \mathrm{H}^{-1} \mathrm{H}$ dipolar couplings from $n$-alkanes dissolved in a nematic LC KODAK EK $11650^{20}$ (study I), (2) ${ }^{2} \mathrm{H}$ NMR quadrupolar splittings ${ }^{13}$ from dilute solutions of $n$-decane/4'-methoxybenzylidene-4- $n$-butylaniline (MBBA) and 1,6-dimethoxyhexane (1,6-DMH)/MBBA systems (study II), ${ }^{23}$ and (3) deuterium quadrupolar splittings from lyotropic liquid crystalline phases of a ternary system of sodium octanoate, 1 -decanol, and water. ${ }^{24}$

\footnotetext{
${ }^{\dagger}$ To whom correspondence should be addressed (Phone: 043-290-3394, Fax: 043-290-3395, E-mail: sasanuma@planet.tc.chiba-u.ac.jp).
} 
In this paper, the simulation scheme and results of study I are explained in detail. In the next paper, ${ }^{23}$ as study II, conformational characteristics of 1,6-DMH are to be compared with those of $n$-decane, and the orientations of the solutes and solvent will be described as functions of temperature and concentration. The methodology proposed here is shown valid for conformational analysis of chain molecules incorporated in anisotropic fields.

\section{MODEL}

\section{Rotational Isomeric State (RIS) Approximation}

Conformations of chain molecules in the free state may be represented only in terms of short-range intramolecular interactions. The RIS scheme, ${ }^{1}$ developed mostly for polymeric chains, is based on this hypothesis. The weights of all possible conformations may be evaluated from statistical weight matrixes, $U_{n} \mathrm{~s}$ ( $n$ : bond number), assigned to skeletal bonds. When intramolecular interactions up to the second-order interactions are taken into account, relative weights of all conformations may be expressed as functions of only two parameters, $\sigma$ and $\omega$. Weights are defined as the Boltzmann factor of the corresponding conformational energy; e.g., $\sigma=\exp$ ( $\left.-E_{\sigma} / R T\right)$, where $R$ is the gas constant and $T$ is the absolute temperature. $U_{n}$ matrixes of $n$-alkanes are given by $^{1,2}$

$$
U_{2}=\left(\begin{array}{ccc}
1 & \sigma & \sigma \\
0 & 0 & 0 \\
0 & 0 & 0
\end{array}\right)
$$

and

$$
U_{n}=\left(\begin{array}{ccc}
1 & \sigma & \sigma \\
1 & \sigma & \sigma \omega \\
1 & \sigma \omega & \sigma
\end{array}\right)(n \geq 3)
$$

Here, the rows and columns of the matrixes are indexed to rotational states for the previous and current bonds. Fractional population $f_{\xi \eta \zeta} \ldots$ of a conformation $\xi \eta \zeta \ldots$ $\left(\xi, \eta, \zeta=t, g^{+}\right.$, or $\left.g^{-}\right)$may be calculated as

$$
f_{\xi \eta \zeta} \ldots=\frac{\mathbf{J}^{*}\left[U_{2}(\xi) U_{3}^{\prime}(\xi \eta) U_{4}^{\prime}(\eta \zeta) \ldots\right] \mathbf{J}}{\mathbf{J}^{*}\left[\prod_{n=2}^{N-1} U_{n}\right] \mathbf{J}}
$$

where $\mathbf{J}^{*}=[100], \mathbf{J}$ is the $3 \times 1$ column matrix of which elements are unity, and $N$ is the number of skeletal bonds. The $U_{2}(\xi)$ matrix may be obtained by filling the columns of the $U_{2}$ matrix other than that corresponding to the $\xi$ state with zero, and the $U_{3}^{\prime}(\xi \eta)$ matrix by filling the elements of the $U_{3}$ matrix other than that corresponding to the $\xi \eta$ conformation with zero, etc.

If $n$-alkanes are placed in nematic fields, i.e., in a flexible cylindrical potential well, conformations must be perturbed from the free state. By minor revision, the RIS scheme may be applicable for evaluation of conformer populations of such perturbed molecules. The $\mathrm{C}-\mathrm{C}$ bonds are expected to have different degrees of the rotational freedom. Thus, statistical weights of individual $\mathrm{C}-\mathrm{C}$ bonds must be distinguished:
Table I. Geometrical parameters of $n$-alkanes ${ }^{\text {a }}$

Bond length $/ \AA$
C-H

$\begin{array}{ll}\mathrm{C}-\mathrm{C} & 1.529\end{array}$

Bond angle/degree

$\angle \mathrm{HCH}$ of methyl group $\quad 107.7$

$\angle \mathrm{HCC}$ of methyl group $\quad 111.2$

$\angle \mathrm{HCC}$ of methylene group $\quad 109.3$

$\angle \mathrm{CCC}$

Dihedral angle for gauche state, degree bond 2

bonds $n(\geq 3)$

Van der Waals radius/ $\AA$

$$
\mathrm{H}
$$

C

${ }^{a}$ Determined for $n$-hexane by geometrical optimization using $a b$ initio molecular orbital calculations at the $\mathrm{HF} / 6^{--} 31 \mathrm{G}^{*}$ level.

$$
U_{2}=\left(\begin{array}{ccc}
1 & \sigma_{2}^{\prime} & \sigma_{2}^{\prime} \\
0 & 0 & 0 \\
0 & 0 & 0
\end{array}\right)
$$

and

$$
U_{n}=\left(\begin{array}{ccc}
1 & \sigma_{n}^{\prime} & \sigma_{n}^{\prime} \\
1 & \sigma_{n}^{\prime} & \sigma_{n}^{\prime} \omega_{n}^{\prime} \\
1 & \sigma_{n}^{\prime} \omega_{n}^{\prime} & \sigma_{n}^{\prime}
\end{array}\right)(n \geq 3)
$$

where $\sigma_{n}^{\prime}$ and $\omega_{n}^{\prime}$ represent statistical weights including effects of the nematic field. In this modified RIS scheme, all possible molecular symmetries are taken into account, and conformer populations can be calculated from eq 3 using a small number of parameters: $\sigma_{n}^{\prime} \mathrm{s}$ and $\omega_{n}^{\prime} \mathrm{s}$ $(n=2,3, \ldots N-1)$. No a priori assumptions on conformational (internal) energies or potential functions of the nematic field are required.

\section{Orientational Order Parameters}

Orientational order parameters were derived from molecular shape. The principal axes of inertia, determined for each conformer, are assumed coincident with those of the ordering matrix. The shape of the conformer $k$ is approximated by a rectangular parallelepiped with dimensions of $A_{X_{\mathrm{k}}}, A_{Y_{\mathrm{k}}}$, and $A_{Z_{\mathrm{k}}}\left(A_{Z_{\mathrm{k}}} \geq A_{X_{\mathrm{k}}} \geq A_{Y_{\mathrm{k}}}\right){ }^{25} X, Y$, and $Z$ directions are parallel to the principal axes of inertia. The parallelepiped is set so as to just fit each conformer with a van der Waals volume (Table I) ${ }^{26}$ For evaluation of order parameters, the 1980 model of Samulski ${ }^{27}$ was modified and used. The order parameter $S_{Z Z_{k}}$ of a conformer $k$ is assumed to be

$$
S_{Z Z_{k}}=C(T, c)\left(\frac{A_{Z_{k}}}{A_{X_{k}}+A_{Y_{k}}}-\frac{1}{2}\right)
$$

where $C(T, c)$ is the measure of orientational ordering due to the nematic field and depends on solute-solvent interaction as well as temperature $T$ and solute concentration $c$. Biaxiality is assumed to be given by

$$
\frac{S_{X X_{k}}-S_{Y Y_{k}}}{S_{Z Z_{k}}}=\frac{A_{X_{k}}-A_{Y_{k}}}{A_{Z_{k}}}
$$

In Samulski's original model, ${ }^{27}$ the conformer was ap- 
proximated as an ellipsoid. The orientational order parameters were defined using the ellipsoid semiaxes $\alpha_{X}$, $\alpha_{Y}$, and $\alpha_{Z}$, instead of $A_{X_{k}}, A_{Y_{k}}$, and $A_{Z_{k}}$. Here $\alpha_{X}$ is estimated from the principal moments of inertia, $I_{X X}, I_{Y Y}$, and $I_{Z Z}$, according to $\alpha_{X}=\left[\left(I_{Y Y}+I_{Z Z}-I_{X X}\right) \frac{5}{2 M}\right]$, where $M$ is molecular weight. To clarify shape and anisotropy in real space, the parallelepiped approximation was adopted.

\section{${ }^{1} \mathrm{H}^{-1} \mathrm{H}$ Dipolar Coupling}

Observed dipole coupling $D_{i j}$ between two protons i and $\mathrm{j}$ of the solute can be expressed as ${ }^{16,17,20}$

$$
D_{i j}=\sum_{k}^{K} f_{k} D_{i j, k}
$$

where $K$ is the number of conformers. The sum of $f_{k} \mathrm{~s}$ is equal to unity: $\sum_{k}^{K} f_{k}=1$. Dipolar coupling $D_{i j, k}$ of the conformer $k$ is given as ${ }^{4,28}$

$$
\begin{array}{r}
D_{i j, k}=-\frac{\gamma_{\mathrm{H}}^{2} h^{2}}{8 \pi r_{i j, k}^{3}}\left\{S_{\mathrm{ZZ}}\left(3 \cos ^{2} \theta_{\mathrm{Z}, i j, k}-1\right)+\left(S_{X X_{k}}-S_{Y Y_{k}}\right)\right. \\
\left.\times\left(\cos ^{2} \theta_{X, i j, k}-\cos ^{2} \theta_{Y, i j, k}\right)\right\}
\end{array}
$$

where $\gamma_{\mathrm{H}}$ is the gyromagnetic ratio of proton, $h$ is the Planck constant, and, e.g., $\theta_{Z, i j, k}$ is the angle between the $Z$ axis and internuclear vector $\mathbf{r}_{i j, k}$ of the conformer $k$.

\section{Maximum Entropy Method}

The MaxEnt method is useful to derive reliable results from a restricted number of data. ${ }^{21,22}$ Conformer probability of the solute must satisfy the reproducibility of the experimental observations. Agreement between theory and experiment may be monitored by

$$
\chi^{2}=\sum_{i j} \frac{\left(D_{i j, \text { calc }}-D_{i j, \text { obsd }}\right)^{2}}{\varepsilon_{i j}^{2}}
$$

where $D_{i j \text {,calc }}$ and $D_{i j, \text { obsd }}$ are, respectively, calculated and observed dipolar couplings, and $\varepsilon_{i j}$ is the experimental error.

Entropy of the conformer probabilities is ${ }^{29}$

$$
S\left(f_{k}\right)=\sum_{k=1}^{K}\left(f_{k}-m_{k}-f_{k} \ln \frac{f_{k}}{m_{k}}\right)
$$

where $m_{k}$ is the initial model of $f_{k}$. In the MaxEnt method, the most probable solution of $f_{k}$ s may maximize $S$. Accordingly, the solution may be found at maximum of

$$
Q=\alpha S-\frac{\chi^{2}}{2}
$$

where $\alpha$ is the regularization constant, which counterbalances the two requirements: maximizing of entropy and reproduction of experiment. Entropy $S$ is convex and $\chi^{2}$ is concave, and thus $Q$ always reaches a unique maximum. MaxEnt simulation is carried out under the restriction of the RIS approximation.

The MaxEnt method has been incorporated into meanfield theories for anisotropic fields and applied to conformational analysis of flexible molecules dissolved in nematic solvents. ${ }^{30-32}$ This method has been modified for simultaneous analysis of different types of experi- (a)

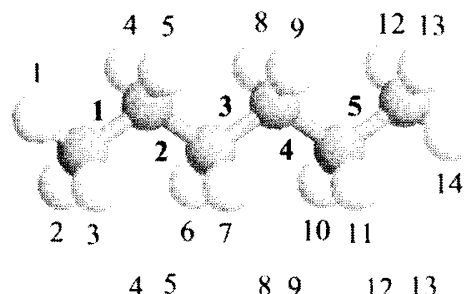

(b)

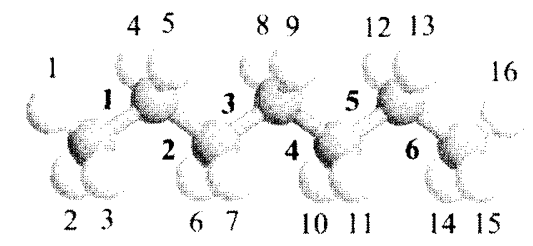

(c)

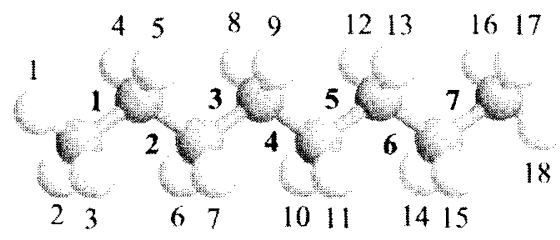

(d)

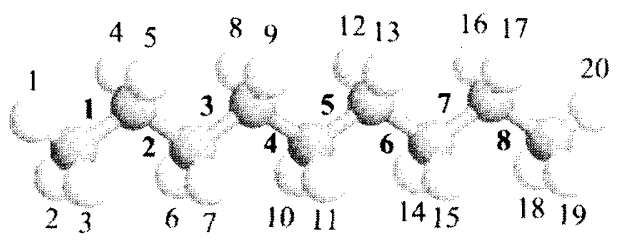

(e)

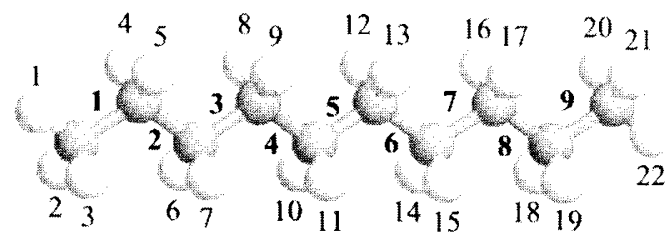

Figure 1. Schematic representation of $n$-alkanes in all-trans conformation: (a) $n$-hexane; (b) $n$-heptane; (c) $n$-octane; (d) $n$-nonane; (e) $n$-decane. Proton atoms and $\mathrm{C}-\mathrm{C}$ bonds are numbered as indicated.

mental data from the flexible solutes. ${ }^{33}$ However, our approach is based on the RIS scheme, and the MaxEnt method was adopted for parameter optimization to acquire the most probable solution from a restricted number of observations.

\section{Procedure for Simulation}

Based on the above, the simulation proceeds as follows: (Step 1) All possible conformations are enumerated within the framework of RIS approximation. Conformer fractions are calculated from eq 3 using a given set of statistical weight parameters $\sigma_{n}^{\prime} \mathrm{s}$ and $\omega_{n}^{\prime} \mathrm{s}$. Entropy $S$ for conformer fractions is estimated according to eq 11 using a proper initial model $m_{k}$ s. (Step 2) For each conformer, the principal axes of inertia are determined from geometrical parameters, and the dimensions $A_{X_{k}}, A_{Y_{k}}$, and $A_{Z_{k}}$ of the conformer are evaluated. Orientational order parameters $S_{Z Z_{k}}$ and $S_{X X_{k}}-S_{Y Y_{k}}$ are calculated from eqs 6 and 7. (Step 3) ${ }^{1} \mathrm{H}-{ }^{1} \mathrm{H}$ dipolar couplings are calculated from eqs 8 and 9 , and compared with experimental data to obtain $\chi^{2}$. Iterative computations of Steps 1 to 3 are carried out using a new set of variables $\sigma_{n}^{\prime} \mathrm{s}, \omega_{n}^{\prime} \mathrm{s}$, and $C(T, c)$ determined so as to increase $Q$ efficiently, until the $Q$ function is maximized within a given allowance. 
Table II. Calculated and observed values of dipolar couplings $n$-hexane

\begin{tabular}{|c|c|c|}
\hline \multirow{2}{*}{$\begin{array}{c}\text { Proton pair }^{\mathrm{a}} \\
i-j\end{array}$} & \multicolumn{2}{|c|}{$D_{i j} / \mathrm{Hz}$} \\
\hline & Calculated & Observed \\
\hline $1-2$ & 1557 & 1526 \\
\hline $4-5$ & 3202 & 3288 \\
\hline $6-7$ & 3744 & 3711 \\
\hline $1-4$ & -275 & -310 \\
\hline $1-6$ & -841 & -852 \\
\hline $1-8$ & -515 & -484 \\
\hline $1-10$ & -260 & -272 \\
\hline $1-12$ & -163 & -167 \\
\hline $4-8$ & -1393 & -1323 \\
\hline $4-9$ & -895 & -881 \\
\hline $\mathrm{RMSE}^{\mathrm{b}} / \mathrm{H}$ & \multicolumn{2}{|c|}{41} \\
\hline
\end{tabular}

${ }^{\mathrm{a}}$ For proton numbers $i$ and $j$, see Figure 1. ${ }^{\mathrm{b}}$ Defined in eq 13.

Table III. Calculated and observed values of dipolar couplings for $n$-heptane

\begin{tabular}{|c|c|c|}
\hline \multirow{2}{*}{$\begin{array}{c}\text { Proton pair }{ }^{\mathrm{a}} \\
i \cdot j\end{array}$} & \multicolumn{2}{|c|}{$D_{i j} / \mathrm{Hz}$} \\
\hline & Calculated & Observed \\
\hline $1-2$ & 1517 & 1489 \\
\hline $4-5$ & 3369 & 3461 \\
\hline $6-7$ & 3893 & 3897 \\
\hline $8-9$ & 4123 & 4093 \\
\hline $1-4$ & -253 & -226 \\
\hline $1-6$ & -857 & -916 \\
\hline $1-8$ & -511 & -520 \\
\hline $1-10$ & -275 & -290 \\
\hline $1-12$ & -185 & -192 \\
\hline $1-14$ & -115 & -127 \\
\hline $4-8$ & -1436 & -1453 \\
\hline $4-9$ & -927 & -924 \\
\hline $6-10$ & -1538 & -1575 \\
\hline $6-11$ & -975 & -975 \\
\hline $\operatorname{RMSE}^{b} / 1$ & \multicolumn{2}{|c|}{34} \\
\hline
\end{tabular}

${ }^{\mathrm{a}}$ For proton numbers $i$ and $j$, see Figure 1. ${ }^{\mathrm{b}}$ Defined in eq 13.

For simulation, the MemSys5 package ${ }^{34}$ from the Maximum Entropy Data Consultants Ltd. was made to combine with the RIS scheme. Adjustment of the regulation constant $\alpha$ and calculations of $Q$ and $S$ are based on the original algorism of MemSys5. ${ }^{35}$

\section{RESULTS AND DISCUSSION}

\section{Geometrical Parameters}

Geometrical parameters determined from the $a b$ initio molecular orbital (MO) calculations for $n$-hexane at the Hartree-Fock level of theory using the $6-31 \mathrm{G}^{*}$ basis set $\left(\mathrm{HF} / 6-31 \mathrm{G}^{*}\right)^{36,37}$ were used for all $n$-alkanes here. The values are listed in Table I. The dihedral angle for the trans state was set equal to $0^{\circ}$. Dihedral angles for the gauche states of the bonds 2 and $n(\geq 3)$ were based on those of the gtt and tgt conformers of $n$-hexane. The van der Waals radii of carbon and hydrogen atoms were taken from Bondi's table. ${ }^{26}$

\section{Analysis}

The statistical weight parameter $\omega_{n}^{\prime}$ represents the frequency of $\mathrm{g}^{ \pm} \mathrm{g}^{\mp}$ conformations at successive $n-1$ and
Table IV. Calculated and observed values of dipolar couplings for $n$-octane

\begin{tabular}{ccc}
\hline Proton pair $^{a}$ & \multicolumn{2}{c}{$D_{i j} / \mathrm{Hz}$} \\
\cline { 2 - 3 }$i-j$ & Calculated & Observed \\
\hline $1-2$ & $\mathbf{1 6 5 6}$ & 1637 \\
$4-5$ & 3479 & 3539 \\
$6-7$ & 4050 & 4119 \\
$8-9$ & 4363 & 4319 \\
$1-4$ & -292 & -319 \\
$1-6$ & -912 & -949 \\
$1-8$ & -510 & -538 \\
$1-10$ & -288 & -296 \\
$1-12$ & -205 & -204 \\
$1-14$ & -135 & -129 \\
$1-16$ & -89 & -96 \\
$4-8$ & -1542 & -1544 \\
$4-9$ & -961 & -976 \\
$6-10$ & -1637 & -1710 \\
$6-11$ & -1025 & -1088 \\
& &
\end{tabular}

$\operatorname{RMSE}^{\mathrm{b}} / \mathrm{Hz}$

39

\footnotetext{
${ }^{\mathrm{a}}$ For proton numbers $i$ and $j$, see Figure 1. ${ }^{\mathrm{b}}$ Defined in eq 13.
}

Table V. Calculated and observed values of dipolar couplings for $n$-nonane

\begin{tabular}{|c|c|c|}
\hline \multirow{2}{*}{$\begin{array}{c}\text { Proton pair }{ }^{\mathrm{a}} \\
i \cdot j\end{array}$} & \multicolumn{2}{|c|}{$D_{\mathrm{ij}} / \mathrm{Hz}$} \\
\hline & Calculated & Observed \\
\hline $1-2$ & 1574 & 1546 \\
\hline $4-5$ & 3451 & 3570 \\
\hline $6-7$ & 3997 & 4104 \\
\hline $8-9$ & 4192 & 4420 \\
\hline $10-11$ & 4089 & 4220 \\
\hline $1-4$ & -264 & -265 \\
\hline $1-6$ & -915 & -958 \\
\hline $1-8$ & -457 & -539 \\
\hline $1-10$ & -267 & -300 \\
\hline $1-12$ & -196 & -206 \\
\hline $1-14$ & -133 & -138 \\
\hline $1-16$ & -93 & -93 \\
\hline $1-18$ & -64 & -73 \\
\hline $4-8$ & -1580 & -1554 \\
\hline $4-9$ & -942 & -977 \\
\hline $6-10$ & -1640 & -1721 \\
\hline $6-11$ & -970 & -1028 \\
\hline $8-12$ & -1647 & -1779 \\
\hline $8-13$ & -960 & -1097 \\
\hline $\mathrm{RMSE}^{\mathrm{b}} / \mathrm{Hz}$ & \multicolumn{2}{|c|}{90} \\
\hline
\end{tabular}

${ }^{\mathrm{a}}$ For proton numbers $i$ and $j$, see Figure $1 . \quad{ }^{\mathrm{b}}$ Defined in eq 13.

$n$ bonds of the alkane chain. Even in the free state, these conformational sequences require as much as $3.0 \mathrm{kcal}$ $\mathrm{mol}^{-1}\left(=2 E_{\sigma}+E_{w}\right)$ relative to the all-trans state. ${ }^{1,2}$ Since this must rarely occur in a uniaxial environment, $\omega_{n}^{\prime}$ should be very small. To reduce the variables and facilitate simulation, all $\omega$ were assumed equal, i.e., that the frequency of the chain folding does not depend on position. This hypothesis appears crude, because the central folding may more distort conformer shape and more disturb orientational orderings of solute and solvent than the terminal folding.

On the above assumption, parameters $C(T, c), \sigma_{n}^{\prime}$ 's and $\omega^{\prime}$ are treated as variables. All conformers within the framework of the RIS approximation were used: $27(n$-hexane), $81(n$-heptane), 243( $n$-octane), $729(n$ - 
Table VI. Calculated and observed values of dipolar couplings for $n$-decane

\begin{tabular}{ccc}
\hline Proton pair $^{\mathrm{a}}$ & \multicolumn{2}{c}{$D_{i j} / \mathrm{Hz}$} \\
\cline { 2 - 3 }$i-j$ & Calculated & Observed \\
\hline $1-2$ & 1712 & 1669 \\
$4-5$ & 3608 & 3660 \\
$6-7$ & 4218 & 4283 \\
$8-9$ & 4559 & 4577 \\
$10-11$ & 4689 & 4697 \\
$1-4$ & -315 & -313 \\
$1-6$ & -931 & -998 \\
$1-8$ & -490 & -556 \\
$1-10$ & -280 & -319 \\
$1-12$ & -198 & -219 \\
$1-14$ & -142 & -151 \\
$1-16$ & -102 & -127 \\
$1-18$ & -76 & -82 \\
$1-20$ & -54 & -57 \\
$4-8$ & -1705 & -1666 \\
$4-9$ & -967 & -1020 \\
$6-10$ & -1856 & -1863 \\
$6-11$ & -1062 & -1062 \\
$8-12$ & -1885 & -1970 \\
$8-13$ & -1078 & -1140 \\
& &
\end{tabular}

$\operatorname{RMSE}^{b} / \mathrm{Hz}$

43

${ }^{\mathrm{a}}$ For proton numbers $i$ and $j$, see Figure 1. ${ }^{\mathrm{b}}$ Defined in eq 13.

Table VII. Optimized parameters

\begin{tabular}{lccccc}
\hline & $n$-Hexane & $n$-Heptane & $n$-Octane & $n$-Nonane & $n$-Decane \\
\hline$C(T, c)$ & 0.431 & 0.406 & 0.409 & 0.373 & 0.392 \\
$\sigma_{2}^{\prime}$ & 0.355 & 0.295 & 0.231 & 0.193 & 0.189 \\
$\sigma_{3}^{\prime}$ & 0.453 & 0.365 & 0.324 & 0.226 & 0.201 \\
$\sigma_{4}^{\prime}$ & & & 0.412 & 0.374 & 0.311 \\
$\sigma_{5}^{\prime}$ & & & & & 0.393 \\
$\omega^{\prime}$ & 0.000 & 0.000 & 0.000 & 0.000 & 0.000 \\
\hline
\end{tabular}

Table VIII. Orientational order parameters of $n$-alkanes

\begin{tabular}{lcc}
\hline & $\left\langle S_{Z Z}\right\rangle$ & $\left\langle S_{X X}-S_{Y Y}\right\rangle$ \\
\hline$n$-Hexane & 0.185 & 0.0106 \\
$n$-Heptane & 0.202 & 0.0127 \\
$n$-Octane & 0.229 & 0.0137 \\
$n$-Nonane & 0.231 & 0.0156 \\
$n$-Decane & 0.258 & 0.0205 \\
\hline
\end{tabular}

nonane), and 2187 ( $n$-decane). To investigate the dependence of the results on initial $m_{k}$, two sets of initial data were adopted: (1) uniform population, i.e., $m_{k}=1 / K$ and (2) free state population, i.e., the $m_{k}$ value calculated from eqs $1-3$ using $E_{\sigma}=0.5$ and $E_{\omega}=2.0 \mathrm{kcal} \mathrm{mol}^{-1}$. Although the latter always gave more rapid convergence than the former, both data provided essentially the same results in all simulations.

In Tables II-VI, calculated ${ }^{1} \mathrm{H}-{ }^{1} \mathrm{H}$ dipolar couplings of the $n$-alkanes are compared with the corresponding experimental data. Reproducibility may be given by the root-mean-square error (RMSE)

$$
\mathrm{RMSE}=\left[\sum_{i j}^{L} \frac{\left(D_{i j, \mathrm{obsd}}-D_{i j, \mathrm{calc}}\right)^{2}}{L}\right]^{\frac{1}{2}}
$$

where $L$ is the number of experimental data. RMSEs for $n$-hexane, $n$-heptane, $n$-octane, $n$-nonane, and $n$-decane were obtained as $41,34,39,90$, and $43 \mathrm{~Hz}$, and the experimental error $\varepsilon_{i j}$ was reported to be $\pm 25 \mathrm{~Hz} .{ }^{20}$ Except
Table IX. Conformer probabilities of $n$-alkanes

\begin{tabular}{|c|c|c|c|c|c|}
\hline & \multirow{2}{*}{$\begin{array}{l}\text { Total no. of } \\
\text { conformers }\end{array}$} & \multirow{2}{*}{$\begin{array}{l}\text { No. of } \\
\text { gache } \\
\text { bonds }\end{array}$} & \multirow{2}{*}{$\begin{array}{c}\text { No. of } \\
\text { conformers }\end{array}$} & \multicolumn{2}{|c|}{ Probabilities } \\
\hline & & & & in $\mathrm{LC}$ & $\begin{array}{c}\text { Free } \\
\text { state }^{a}\end{array}$ \\
\hline \multirow[t]{4}{*}{$n$-Hexane } & 27 & 0 & 1 & 0.22 & 0.18 \\
\hline & & 1 & 6 & 0.51 & 0.49 \\
\hline & & 2 & 12 & 0.25 & 0.30 \\
\hline & & 3 & 8 & 0.02 & 0.03 \\
\hline \multirow[t]{5}{*}{$n$-Heptane } & 81 & 0 & 1 & 0.14 & 0.11 \\
\hline & & 1 & 8 & 0.44 & 0.38 \\
\hline & & 2 & 24 & 0.34 & 0.38 \\
\hline & & 3 & 32 & 0.07 & 0.12 \\
\hline & & 4 & 16 & 0.01 & 0.01 \\
\hline \multirow[t]{6}{*}{$n$-Octane } & 243 & 0 & 1 & 0.11 & 0.06 \\
\hline & & 1 & 10 & 0.37 & 0.28 \\
\hline & & 2 & 40 & 0.37 & 0.40 \\
\hline & & 3 & 80 & 0.13 & 0.22 \\
\hline & & 4 & 80 & 0.02 & 0.04 \\
\hline & & 5 & 32 & 0.00 & 0.00 \\
\hline \multirow[t]{7}{*}{$n$-Nonane } & 729 & 0 & 1 & 0.08 & 0.04 \\
\hline & & 1 & 12 & 0.32 & 0.20 \\
\hline & & 2 & 60 & 0.37 & 0.36 \\
\hline & & 3 & 160 & 0.17 & 0.29 \\
\hline & & 4 & 240 & 0.06 & 0.10 \\
\hline & & 5 & 192 & 0.00 & 0.01 \\
\hline & & 6 & 64 & 0.00 & 0.00 \\
\hline \multirow[t]{8}{*}{$n$-Decane } & 2187 & 0 & 1 & 0.06 & 0.02 \\
\hline & & 1 & 14 & 0.27 & 0.14 \\
\hline & & 2 & 84 & 0.36 & 0.31 \\
\hline & & 3 & 280 & 0.23 & 0.32 \\
\hline & & 4 & 560 & 0.07 & 0.17 \\
\hline & & 5 & 672 & 0.01 & 0.04 \\
\hline & & 6 & 448 & 0.00 & 0.00 \\
\hline & & 7 & 128 & 0.00 & 0.00 \\
\hline
\end{tabular}

${ }^{\mathrm{a}}$ Calculated using conformational energies of $E_{\sigma}=0.5$ and $E_{\omega}=$ $2.0 \mathrm{kcal} \mathrm{mol}^{-1}$ for a reduced temperature $T^{*}=0.875\left(T^{*}=T / T_{\mathrm{NI}}\right)$.

for $n$-nonane, agreement between theory and experiment is thus satisfactory.

In Table VII, optimized values of variables are listed. $\omega^{\prime}$ was found to be null for all compounds, indicating even short chains such as $n$-hexane can hardly have $\mathrm{g}^{ \pm} \mathrm{g}^{\mp}$ sequences in the nematic field. If $n$-alkanes had such folding, as chain length increases, the nematic order should be more disturbed by the defect. In other words, a longer $n$-alkane chain should not also have the conformational sequences. Consequently, the above simplification that all $\omega_{n}^{\prime}$ s are set to the same value is justified.

Orientational Order, Conformation, and Shape of Solutes

In Table VIII, averaged orientational order parameters of the solutes are listed as calculated from

$$
\left\langle S_{Z Z_{k}}\right\rangle=\sum_{k}^{K} S_{Z Z_{k}} f_{k}
$$

and

$$
\left\langle S_{X X}-S_{Y Y}\right\rangle=\sum_{k}^{K}\left(S_{X X_{k}}-S_{Y Y_{k}}\right) f_{k}
$$

Since NMR measurements for all $n$-alkanes were performed at the same reduced temperature $T / T_{\mathrm{NI}}$ of 
Table X. Averaged molecular dimensions of $n$-alkanes

\begin{tabular}{|c|c|c|c|c|c|c|c|c|c|c|}
\hline & \multicolumn{2}{|c|}{$\left\langle A_{X}\right\rangle / \AA$} & \multicolumn{2}{|c|}{$\left\langle A_{Y}\right\rangle / \AA$} & \multicolumn{2}{|c|}{$\left\langle A_{Z}\right\rangle / \AA$} & \multicolumn{2}{|c|}{$V^{a} / \AA^{3}$} & \multicolumn{2}{|c|}{$\langle r\rangle^{\mathrm{b}} / \mathrm{A}$} \\
\hline & Nematic & Free state $^{c}$ & Nematic & Free state $^{c}$ & Nematic & Free state ${ }^{c}$ & Nematic & Free state $^{c}$ & Nematic & Free state $^{c}$ \\
\hline$n$-Hexane & 5.56 & 5.61 & 5.00 & 5.04 & 9.69 & 9.64 & 269 & 273 & 5.70 & 5.62 \\
\hline$n$-Heptane & 5.85 & 5.93 & 5.13 & 5.20 & 10.81 & 10.64 & 324 & 328 & 6.75 & 6.59 \\
\hline$n$-Octane & 6.02 & 6.14 & 5.28 & 5.34 & 11.83 & 11.58 & 376 & 380 & 7.78 & 7.51 \\
\hline$n$-Nonane & 6.31 & 6.49 & 5.38 & 5.47 & 12.88 & 12.49 & 437 & 443 & 8.82 & 8.40 \\
\hline$n$-Decane & 6.64 & 6.82 & 5.46 & 5.58 & 13.82 & 13.35 & 501 & 508 & 9.78 & 9.26 \\
\hline
\end{tabular}

$0.875,{ }^{20}$ we may compare order parameters among $n$-alkanes. $\left\langle S_{Z Z}\right\rangle$ increases with chain length.

In Table IX, conformer probabilities of $n$-alkanes are arranged according to the number of gauche bonds and compared with those for the free state. In LC, conformations with a small number of gauche states are more populated than in the free state. Fractions of the alltrans conformation were estimated as $22 \%$ (18\%) for $n$-hexane, $14 \%(11 \%)$ for $n$-heptane, $11 \%(6 \%)$ for $n$ octane, $8 \%(4 \%)$ for $n$-nonane, and $6 \%(2 \%)$ for $n$-decane. The values in the parentheses are those for the free state.

From average dimensions of the rectangular parallelepiped, we may estimate the shape of a solute molecule. Ensemble averages, $\left\langle A_{X}\right\rangle,\left\langle A_{Y}\right\rangle$, and $\left\langle A_{Z}\right\rangle$, can be evaluated from, e.g.,

$$
\langle A X\rangle=\sum_{k}^{K} A_{X_{k}} f_{k}
$$

The average distance between terminal methyl carbons was calculated and is shown in Table X. Differences in $\left\langle A_{X}\right\rangle,\left\langle A_{Y}\right\rangle$, and $\left\langle A_{Z}\right\rangle$ between in LC and in the free state are small, but indicate that nematic constraints somewhat enhance shape anisotropy. This can be seen from the end-to-end distance $\langle r\rangle$. Elongation relative to the free state was evaluated as $1.4 \%$ for $n$-hexane, $2.4 \%$ for $n$-heptane, $3.6 \%$ for $n$-octane, $5.0 \%$ for $n$-nonane, and $5.6 \%$ for $n$-decane. Relative elongation increases with chain length. Interestingly, average volume $V$ of the rectangular parallelepiped was found to be virtually constant.

\section{Comparison with Previous Studies}

${ }^{1} \mathrm{H}^{-1} \mathrm{H}$ dipolar couplings from $n$-hexane were analyzed according to the previous method using SOM approximation. ${ }^{12}$ All conformers were assumed to have the same order parameters. In the simulation, adjustable parameters were $S_{Z Z}, S_{X X}-S_{Y Y}, S_{X Y}, S_{X Z}, S_{Y Z}, \sigma_{2}^{\prime}$, and $\sigma_{3}^{\prime}$, while $\omega_{3}^{\prime}$ was fixed at zero.

RMSE was minimized to $28 \mathrm{~Hz}$ and thus comparable to the experimental error of $25 \mathrm{~Hz}$. This simulation gave better agreement with experiment than the RIS-MaxEnt scheme $(41 \mathrm{~Hz})$, probably due to the difference in the number of variables. The fraction of all-trans conformation of the $n$-hexane was obtained as $0.34(0.22)$, and those of conformers including one, two, and three gauche bonds are $0.50,0.14$, and $0.01(0.51,0.25$, and 0.02$)$, respectively. The values in the parentheses represent the corresponding fractions evaluated here. Our previous analysis suggested that the $n$-hexane molecule is considerably rigid. These results appear consistent with thermodynamic studies, ${ }^{18,19}$ which indicate that $n$-alkanes behave like a chain composed of two complete rigid and additional semiflexible segments at infinite dilution. It should be noted that ${ }^{1} \mathrm{H}^{-1} \mathrm{H}$ dipolar couplings were measured at a solute concentration of as high as $30 \mathrm{~mol} \%$. Thus, it seems reasonable to conclude that the SOM model overestimates populations of anisotropic conformers.

${ }^{1} \mathrm{H}^{-1} \mathrm{H}$ dipolar couplings were analyzed using meanfield theories ${ }^{20}$ based on the elastic continuum model $(0.27),{ }^{10}$ the biaxial-parallelepiped model $(0.28),{ }^{8,9}$ chord representation $(0.20){ }^{11}$ and Monte Carlo sampling $(0.233){ }^{38}$ Recently, the experimental data have also been treated by molecular dynamics simulations $(0.21)^{39}$ and the maximum entropy internal method (0.198). ${ }^{40}$ Here, the values in parentheses represent the all-trans fraction of $n$-hexane, evaluated by the individual models. Conformer populations obtained here are quantitatively comparable to those reported in these studies. It is of interest and significance that our model gives essentially the same results as other theories.

Acknowledgments. The author thanks Professor A. Abe of Tokyo Institute of Polytechnics for helpful advice at the early stage of this study and Dr. S. Tsuzuki of NIMC for providing the MO data on $n$-hexane. This work was supported in part by Grant-in-Aid for Scientific Research (C) (No. 11650920) of Japan Society for the Promotion of Science.

\section{REFERENCES}

1. P. J. Flory, "Statistical Mechanics of Chain Molecules", Interscience, New York, N.Y., 1969.

2. A. Abe, R. L. Jernigan, and P. J. Flory, J. Am. Chem. Soc., 88, 631 (1966).

3. J. W. Emsley, Ed., "Nuclear Magnetic Resonance of Liquid Crystals", Reidel, Dordrecht, 1985.

4. R. Y. Dong, "Nuclear Magnetic Resonance of Liquid Crystals", Springer, New York, N.Y., 1994.

5. See, e.g., G. R. Luckhurst, in "Nuclear Magnetic Resonance of Liquid Crystals", J. W. Emsley, Ed., Reidel, Dordrecht, 1985, Chapter 3; E. E. Burnell and C. A. de Lange, Chem. Rev., 98, 2359 (1998)

6. W. Maier and A. Saupe, Z. Naturforsch., A. Phys. Sci., 14, 882 (1959); 15, 287 (1960).

7. S. Marceljs, J. Chem. Phys., 60, 3599 (1974).

8. G. R. Luckhurst, C. Zannoni, P. L. Nordio, and U. Segre, Mol. Phys., 30, 1345 (1975).

9. J. W. Emsley, G. R. Luckhurst, and C. P. Stockley, Proc. R. Soc. London, Ser. A, 381, 117 (1982).

10. A. J. van der Est, M. Y. Kok, and E. E. Burnell, Mol. Phys., 60, 397 (1987).

11. D. J. Photinos, E. T. Samulski, and H. Toriumi, J. Phys Chem., 94, 4688, 4694 (1990).

12. Y. Sasanuma and A. Abe, Polym. J., 23, 117 (1991)

13. Y. Sasanuma, J. Phys. II France, 3, 1759 (1993). 
14. Y. Sasanuma, J. Phys. II France, 7, 305 (1997).

15. B. Janík, E. T. Samulski, and H. Toriumi, J. Phys. Chem., 91, 1842 (1987).

16. M. Gochin, H. Zimmermann, and A. Pines, Chem. Phys. Lett., 137, 51 (1987).

17. M. Gochin, A. Pines, M. E. Rosen, S. P. Rucker, and C. Schmidt, Mol. Phys., 69, 671 (1990).

18. D. E. Martire, in "The Molecular Physics of Liquid Crystals", G. R. Luckhurst and G. W. Gray, Ed., Academic Press, New York, N.Y., 1979, Chapter 10.

19. B. Kronberg, D. F. Gilson, and D. Patterson, J. Chem. Soc., Faraday Trans. 2, 72, 1673 (1976).

20. M. E. Rosen, S. P. Rucker, C. Schmidt, and A. Pines, J. Phys. Chem., 97, 3858 (1993).

21. R. D. Levine and M. Tribus, Ed., "The Maximum Entropy Formalism," MIT Press, Cambridge, MA, 1979.

22. B. Buck and V. A. Macaulay, Ed., "Maximum Entropy in Action," Oxford University Press, New York, N.Y., 1991.

23. Y. Sasanuma, Polym. J., 32, 890 (2000).

24. A. Suzuki, N. Miura, and Y. Sasanuma, Langmuir, 16, 6317 (2000).

25. J. P. Straley, Phys. Rev. A, 10, 1881 (1974)

26. A. Bondi, J. Phys. Chem., 68, 441 (1964).

27. E. T. Samulski, Ferroelectrics, 30, 83 (1980).

28. P. Diel, in "Nuclear Magnetic Resonance of Liquid Crystals", J. W. Emsley, Ed., Reidel, Dordrecht, 1985, Chapter 7.

29. J. Skilling, in "Maximum Entropy and Bayesian Methods", G.
J. Erickson and C. R. Smith, Ed., Kluwer, Dordrecht, 1988, p 45.

30. R. Berardi, F. Spinozzi, and C. Zannoni, J. Chem. Soc., Faraday Trans., 88, 1863 (1992).

31. J. W. Emsley, I. D. Wallington, D. Catalano, C. Veracini, G. Celebre, and M. Longeri, J. Phys. Chem., 97, 6518 (1993).

32. A. Berardi, F. Spinozzi, and C. Zannoni, Chem. Phys. Lett., 260, 633 (1996).

33. A. Berardi, F. Spinozzi, and C. Zannoni, J. Chem. Phys., 109 3742 (1998).

34. S. F. Gull, M. Charter, and J. Skilling, "MemSys 5 Ver. 1.20", Maximum Entropy Data Consultants Ltd., Cambridge, England, 1991.

35. For the details, see S. F. Gull and J. Skilling, "Quantified Maximum Entropy MemSys5 User's Manual”, Maximum Entropy Data Consultants Ltd., Cambridge, England, 1991.

36. S. Tsuzuki, L. Schaefer, H. Goto, E. D. Jemmis, H. Hosoya, K. Siam, K. Tanabe, and E. Osawa, J. Am. Chem. Soc., 113, 4665 (1991).

37. S. Tsuzuki, Personal communication, 1997

38. M. Luzar, M. E. Rosen, and S. Caldarelli, J. Phys. Chem., 100, 5098 (1996).

39. J. Alejandre, J. W. Emsley, D. J. Tidesley, and P. Carlson, J. Chem. Phys., 101, 7027 (1994).

40. A. Berardi, F. Spinozzi, and C. Zannoni, Chem. Phys. Lett., 260, 633 (1996). 